\title{
Steady Headwind Display with Conditional Angular Rate-Switching Control
}

\author{
Sandip D. Kulkarni, Mark A. Minor, Mark W. Deaver, Eric R. Pardyjak and John M. Hollerbach
}

\begin{abstract}
This research creates a steady headwind at a user position in the scaled Treadport Active Wind Tunnel (TPAWT). The TPAWT adds a wind display system to the previously developed Treadport virtual environment, and this research builds upon prior work to provide improved control of headwind angle at the user position. Key to this research is the addition of a negative pressure plenum at the rear of the treadport to improve nominal flow stability. The previous controller based upon the small gain theorem with a dynamic extension is then modified to provide wind angle feedback control. A conditional angular rate-switching controller is added to reduce wind angle oscillations at the user. A vorticity-meter is developed to assure that the wind flow is centered at the user position. As a result, this research reduces wind angle error by $75 \%$ compared to previous work.
\end{abstract}

${ }^{1}$ Index Terms - Virtual Reality, Haptic Interfaces, Flow Control, Input-output stability, Conditional Angular rate-control.

\section{INTRODUCTION}

$\mathrm{T}$ HIS paper focuses on creating a steady headwind at a user position in the Treadport virtual reality system, Fig. 1. The Treadport allows the user to walk through a virtual environment while viewing realistic renderings on a $180^{\circ}$ CAVE-like display located at the front and sides . To create a highly immersive system for the user, a haptic interface is essential [1]. In [2, 3], the Treadport Active Wind Tunnel (TPAWT) was developed to augment the original Treadport [4] with a wind based haptic interface. Sidewalls, display screens, floor, and ceiling are used to guide airflow supplied through controllable side-vents, Fig 2, to create a pair of counter rotating vortices. A column of airflow then travels between the vortices, which is naturally aimed towards the

Note: This work was supported in part by the National Science Foundation under Grant No. 428856.

S.D. Kulkarni is with the Department of Mechanical Engineering, University of Utah, Salt Lake City, Utah, 84112, and USA. (sandip.kulkarni@utah.edu)

M.A. Minor is with the Department of Mechanical Engineering, University of Utah, Salt Lake City, Utah, 84112, USA (phone: 801-587-7771; fax: 801-585-9826; minor@mech.utah.edu).

M.W. Deaver is with the Department of Mechanical Engineering, University of Utah, Salt Lake City, Utah, 84112, USA.

E.R. Pardyjak is with the Department of Mechanical Engineering, University of Utah, Salt Lake City, Utah, 84112, USA.

J.M. Hollerbach is with the School of Computing, University of Utah, Salt Lake City, Utah, 84112, USA. user. This allows the user to sense air coming towards them as though it is originating from the scenery displayed on the screens. [3] focused on open loop characterization of the flow whereas [2] provided feedback control of wind speed. In contrast to our previous work [2], wind speed control is now feed-forward since we are focusing on wind angle in this research.

In this research, we augment the scaled model TPAWT with a rear-plenum, wind angle controller, and a vorticity

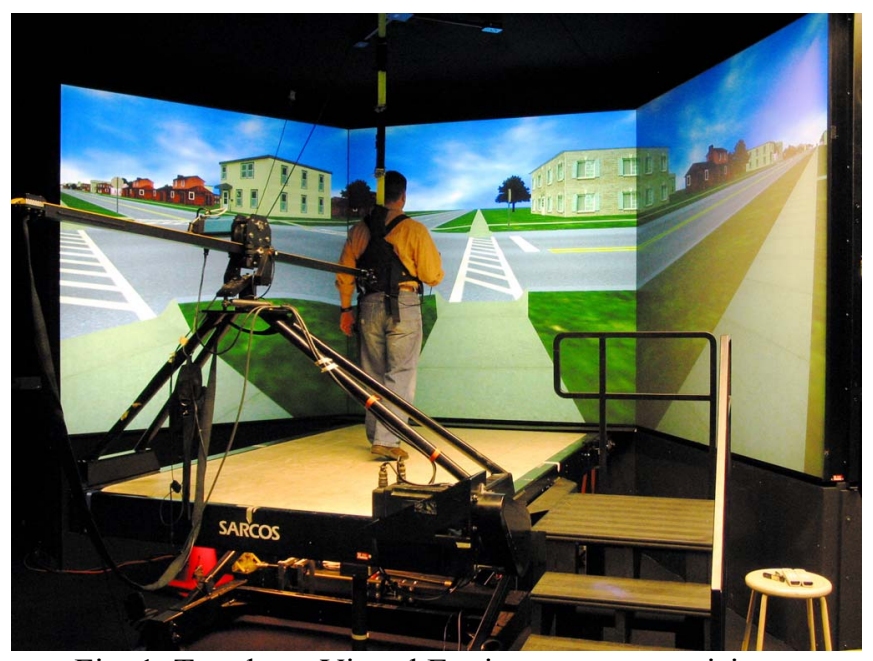

Fig. 1. Treadport Virtual Environment comprising a CAVE-like visual display and locomotion interface

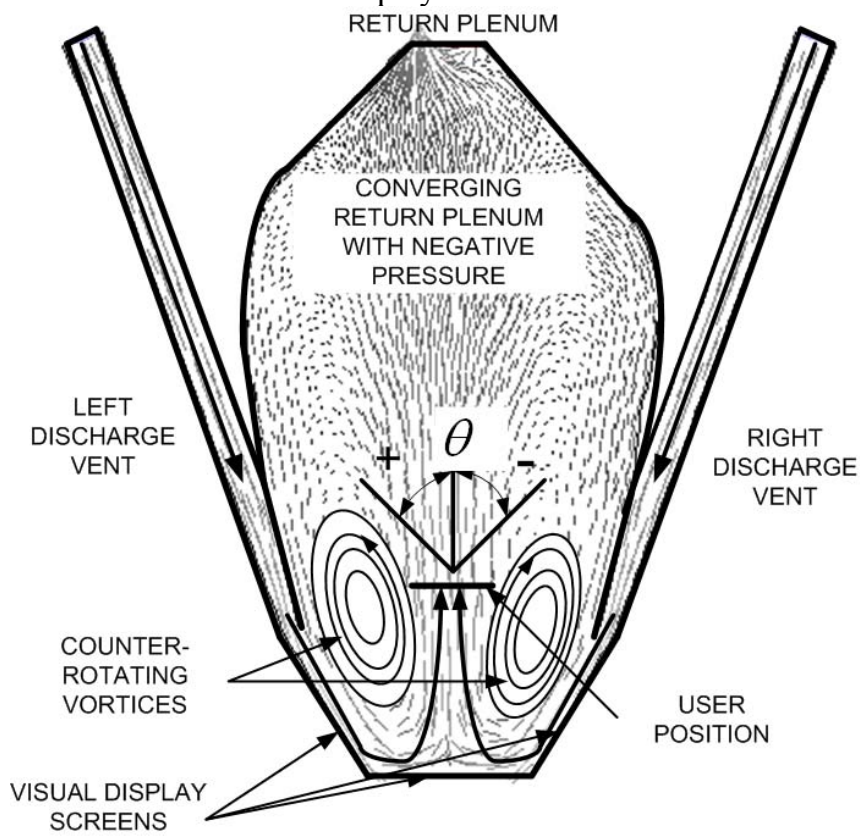

Fig. 2. Stream function plot for input-output characterization. 
meter to determine if the headwind is centered at the user. The return plenum with negative pressure, Fig. 2, was added to the system in $[2,3]$ to stabilize the unsteady wind flow at the user as described in Section V.A. It is a form of predetermined open loop control [5].

With the flow patterns stabilized by the plenum, we can extend the previous feedback controller [2] for wind angle control. Similar to our prior work [2], FLUENT Computational Fluid Dynamics (CFD) analysis also suggests that wind angle at the user is linearly dependent upon the ratio of the inlet vent velocities. These linear relationships are the basis of the CFD derived geometric Jacobian. A dynamic extension combined with the Jacobian forms the core of the feedback controller. However, as vent velocities vary, they feed energy into one of the vortices around the core flow and dissipate it from the other. This makes the vortices dynamically active and oscillations between the vortices can occur. Thus, in Section IV.B we augment the aforementioned controller with conditional angular rate feedback to stabilize the vortices and eliminate oscillations.

The plenum and controller combined together give steady headwind at the user with a nominal $1-3^{\circ}$ deviation. This is approximately a 75\% improvement over prior feed forward wind angle control [2]. As in our prior work, this method is computationally efficient and suited for real-time control, which is a notable contribution relative to ongoing active flow control research.

The paper is organized as follows. Sect. II compares this research to prior work. The Input-Output flow is characterized in Sect. III. The feedback controller is developed in Sect IV and evaluated in Sect V where future work is also discussed. Sect. VI concludes the paper.

\section{BACKGROUND}

Conventionally, the control of fluid flow is approximated by distributed parameter systems based on spatial discretization of partial differential equations. Finite-difference and finite element methods then form systems of ordinary differential equations that serve as a basis for controller design [6-8]. Moreover, controllability, observability, and stability characteristics depend on sensor and actuator locations, discretization method, and a sufficient number of discretization points [9].

Active flow control refers to the manipulation of the system to achieve desired results such as change in drag or flow separation. The Reynolds number for the real TPAWT as well as the experimental setup is $\sim 4 \times 10^{4}$, which indicates that the flow is turbulent and that it can be modeled only approximately. Several researchers have modeled fluid-flow to fit within traditional controllers (linear [10, 11], optimal $[10,12,13]$, and nonlinear $[14,15])$, but there are so many DOF that they are not tractable for real-time control. Thus, control of fluid flow has been studied using reduced order modeling techniques such as Proper Orthogonal Decomposition (POD) [16, 17], Approximate Inertial
Manifolds (AIM) [18], and Eigenmode [19] analysis. Model decomposition methods reduce the DOF to a certain extent, but there still remains a vast gap between the DOF required for real-time active-control of wind flow and the DOF after model decomposition. Moreover change in basis with varying flow patterns must be accounted for. Finally, it should be noted that recent work using Graphics Processing Units (GPU) applied to the QUIC simulation model is capable of predicting and visualizing flow characteristics in real-time for simple flows.

From an alternate perspective, in [20] a LQR controller for thermal processes is developed using distributed parameter control theory and state feedback. This leads to finite dimensional sub-optimal linear output feedback controllers that are similar to our own. Given the complexity of fluid flow models and the requirement for achieving stable flow patterns, this approach is not readily feasible. Rather, the strategy proposed here stabilizes the flow using a combination of predetermined active control, an output feedback algorithms based upon the SGT, and a conditional angular rate-switching feedback control.

The SGT gives a sufficient condition for robust stability of a feedback system so long as the product of the norms of the feedback and plant gains is less than unity. Implementation of the SGT using alternative linear matrix inequality conditions has been applied previously [21]. But, we apply the SGT for input-output control of an infinite dimensional system aided by modified geometry for predetermined active control and conditional angular rate control.

As far as we know, our application of dynamic extension and the small gain theorem combined with predetermined active control and conditional angular rate-switching control is a novel flow control application. Since the method uses an input-output map while considering the system as a black box, it bypasses the problem of access to the model and is readily applicable to real-time control. This paper avoids theoretical aspects of fluid flow control that have limited its practical real-time application.

\section{INPUT-OUTPUT FLOW CHARACTERIZATION}

Steady flow CFD simulations (i.e. time invariant) were initially used to derive geometric Jacobians for feedback control [2]. Subsequent experiments [2, 3], however, indicated that unsteady flow characteristics (i.e. time varying) could not be neglected. In the unsteady flow, the core flow between the vortices is unstable near the desired $\theta=0^{\circ}$ wind angle. Fig. 3 shows a sequence of time lapsed frames taken from an unsteady simulation of the original TPAWT without a return plenum. Note that the vortices have sufficient space to grow, shift and eventually destabilize, regardless of the constant vent velocity ratio. This causes the core flow to push to one side of the TPAWT, acting as a stable but undesirable equilibrium at $\theta= \pm 60^{\circ}$ [3].

The growing, shifting and destabilizing of the two vortices is avoided by modifying the TPAWT geometry. The new geometry is illustrated in Fig. 2, where the rear diffuser 


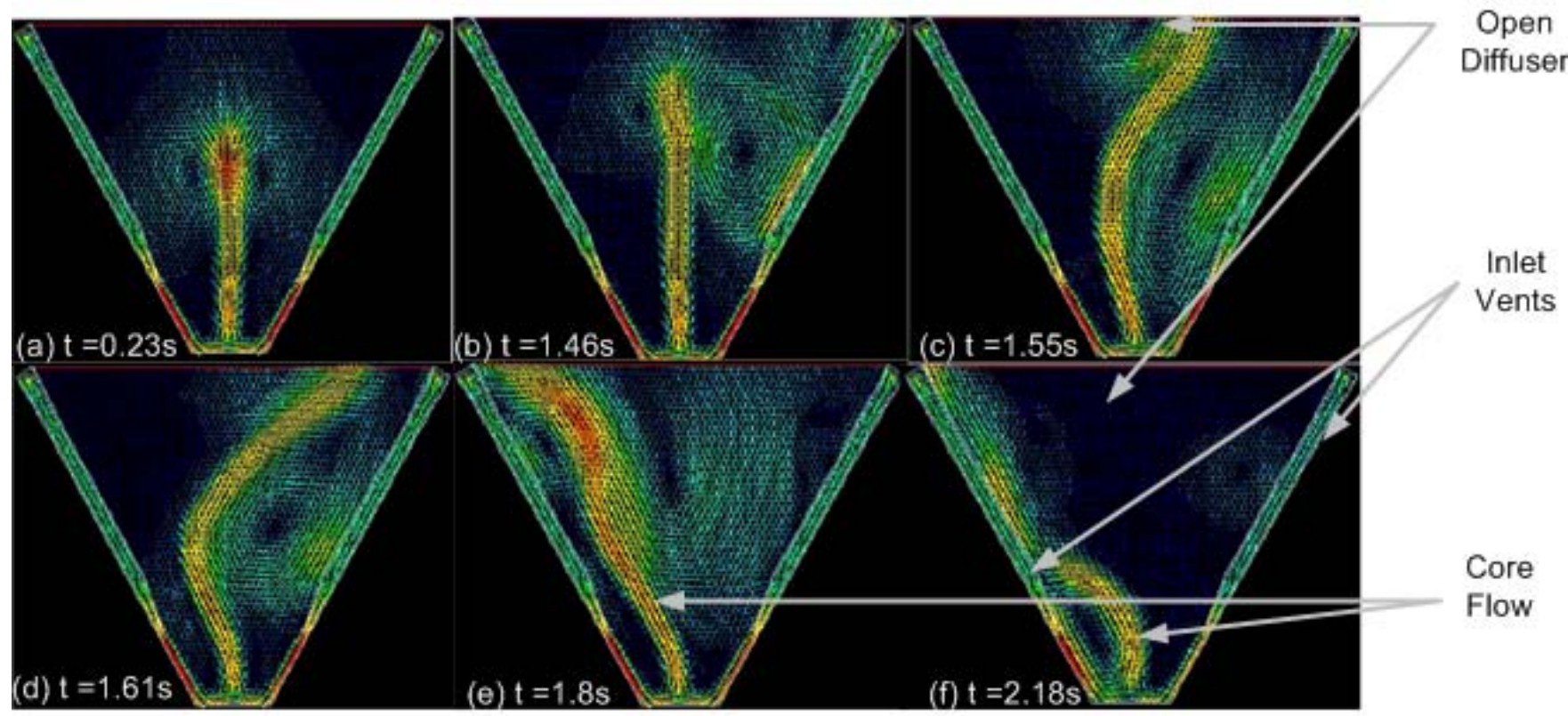

Fig. 3 Stages of vorticity dynamics showing flow instability without the return plenum.

section of the TPAWT is replaced with a negative pressure return-plenum. The stream function plot generated by unsteady FLUENT simulations shown in Fig. 2 indicates the development of a stable head wind at the user. Ideally, if left and right vent velocities, $v_{L}$ and $v_{R}$, respectively, are equal then the vortices are symmetric and the wind angle is $\theta=0^{\circ}$.

Unsteady FLUENT simulations were performed to determine a nominal input-output map for the new system. At ratios far from 1 , the vortices shift the core flow considerably and the core flow is again attracted to one of the stable equilibrium at $\theta= \pm 60^{\circ}$ [3] Hence we consider only the vent velocity ratios in the range from 0.75 to 1.25 . A series of simulations were conducted to sweep this range. At each vent velocity ratio, the flow was allowed to reach steady state and the wind angle was measured to produce Fig. 4.

Using a truncated Taylor series expansion of the output relative to a nominal input, similar to [2], we can evaluate the output change, $\delta \theta$, given an input change, $\delta u$, as,

$$
\delta \theta=\theta\left(u_{0}+\delta u\right)-\theta\left(u_{0}\right)=J_{\theta} \delta u .
$$

where $\delta u$ refers to a change in vent velocity ratio, $v_{L} / v_{R}$. Based on Fig. 4, the geometric Jacobian, $J_{\theta}$, is the slope of regression line shown. This Jacobian is obtained by taking a generalized inverse of the input, as

$$
J_{\theta}=\Delta \theta \Delta u^{-1},
$$

where $\Delta \theta$ is the change in wind angle measured by sensors and $\Delta u$ is change in the ratio of wind velocities at the vents. Thus, the geometric Jacobian is $J_{\theta} \approx-85^{\circ}$

\section{CONTROLler DeVElopment}

The control strategy is based on the combination of passive control provided by the TPAWT geometry and active output feedback control.

\section{A. Output Feedback Controller}

As the results in Section III indicate, the input-output relationship between vent velocity ratio and wind angle is linear. The error states are defined as $e=\theta-\theta_{R}$ where $\theta_{R}$ is the desired output (desired wind angle) and $\theta$ is the actual output wind angle at the user. Taking the time derivative we have,

$$
\dot{e}=\frac{\partial e}{\partial t}=\frac{\partial \theta}{\partial t}-\frac{\partial \theta_{R}}{\partial t} .
$$

Applying the chain rule to (3),

$$
\dot{e}=\frac{\partial y}{\partial u} \frac{\partial u}{\partial t}-\frac{\partial \theta_{R}}{\partial t}
$$

and substituting the geometric Jacobian, $J_{\theta}=\frac{\partial y}{\partial u}$, we then have,

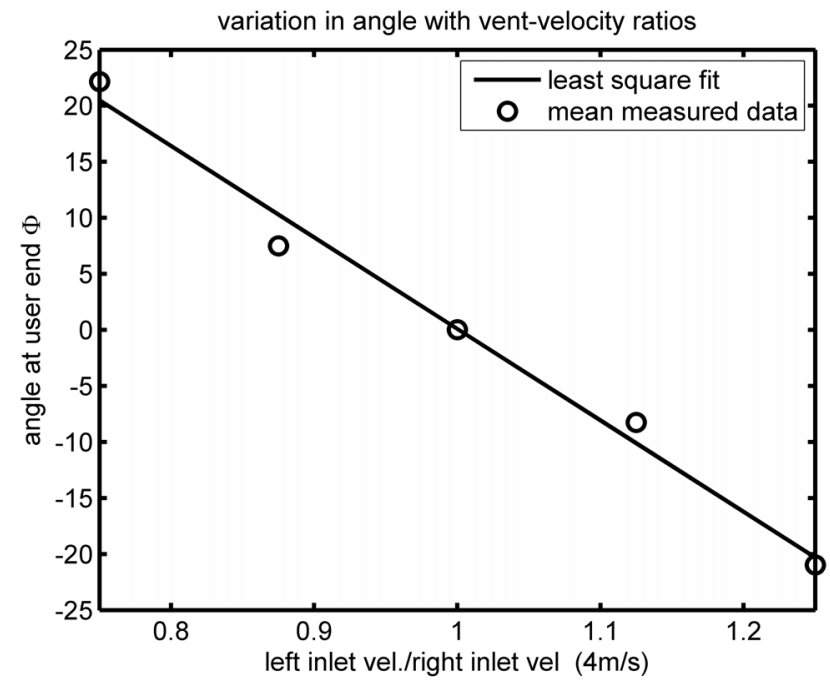

Fig. 4 Wind angle versus vent velocity ratio $u$, based upon unsteady CFD simulations with the return plenum. 


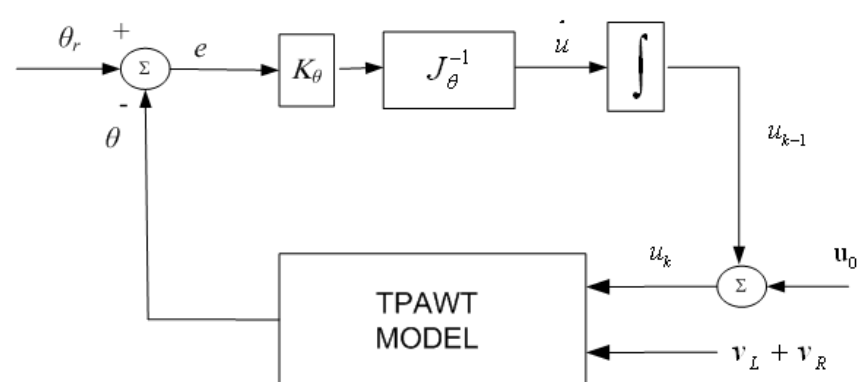

Fig. 5. Equivalent diagram of Small Gain Theorem (SGT) for wind angle control.

$$
\dot{e}=J_{\theta} \dot{u}-\frac{\partial \theta_{R}}{\partial t}=g(e) .
$$

Assigning $g(e)=-K e$, where $K=K^{\mathrm{T}}>0$ is Hurwitz, we can then solve for the desired control inputs,

$$
\dot{u}=J_{\theta}^{-1}\left[K_{\theta} e+\dot{\theta}_{R}\right],
$$

in a fashion similar to backstepping. Assuming regulation only, the resulting control system is then expressed as,

$$
\begin{aligned}
& \dot{e}=-K_{\theta} e=K_{\theta}\left(\theta-\theta_{R}\right) . \\
& \dot{u}=J_{\theta}^{-1} K_{\theta} e
\end{aligned}
$$

Note that the control command $u$ now appears as a state variable. We have applied a dynamic extension to the input. This allows an increase in the relative degree of the system. Eq. (6) also indicates that the geometric Jacobian is coupled with time response as determined by the gain $K$. Unique to this controller is that only the Jacobian $J_{\theta}$ is required for a given operating point.

In order to evaluate stability of the controller, the feedback system (6) can be represented in the form of an interconnected system, Fig. 5, and the Small Gain Theorem (SGT) is applied [2]. Note that the control input is
$u=v_{L} / v_{R}=\int J_{\theta}^{-1} \cdot K_{\theta} e d t$ and the resulting output of the system is $\theta=J_{\theta} u$ where the TPAWT model in Fig. 5 is represented by $J_{\theta}$. Application of the SGT requires that the aforementioned subsystems are finite-gain $L$-stable as described by,

$$
\begin{gathered}
\|u\| \leq \gamma_{1}\|e\|_{L}+\beta_{1} \\
\|\theta\| \leq \gamma_{2}\|u\|_{L}+\beta_{2} .
\end{gathered}
$$

where $\gamma_{1}, \gamma_{2}, \beta_{1}$, and $\beta_{2}$ must all be finite values. Discretizing the subsystems and taking $L$ norms we have,

$$
\begin{gathered}
\left\|u_{k}\right\|_{L} \leq\left\|J_{\theta}^{-1} K_{\theta}\right\|_{L}\left\|e_{k}\right\|_{L} \Delta t+u_{k-1} \\
\left\|\theta_{k}\right\|_{L} \leq\left\|J_{\theta}\right\|_{L}\left\|u_{k}\right\|_{L} \Delta t .
\end{gathered}
$$

From (7)-(10) we have,

$$
\begin{gathered}
\gamma_{1}=\left\|J_{\theta}^{-1}\right\|_{L} \cdot\left\|K_{\theta}\right\|_{L} \cdot \Delta t, \\
\gamma_{2}=\left\|J_{\theta}\right\|_{L}, \\
\beta_{1}=u_{k-1} \quad \beta_{2}=0
\end{gathered}
$$

Considering a discrete system with fixed sampling rates, it is important to note that $\gamma_{1}$ and $\gamma_{2}$ do not change. Furthermore, the SGT requires that $\gamma_{1} \gamma_{2}<1$. Given (11), the feedback connection is finite-gain $L$-stable by the SGT if,

$$
\left\|K_{\theta}\right\|_{L} \Delta t<1 \text {. }
$$

\section{B. Predetermined Active Control}

Besides the factors discussed in Section 3, design asymmetry and unequal delays in the vents can also lead to instability. As shown in Fig. 2, the region of flow separation dominates the formation of vortices. As the vortices become dynamic, the region of separation moves along the display

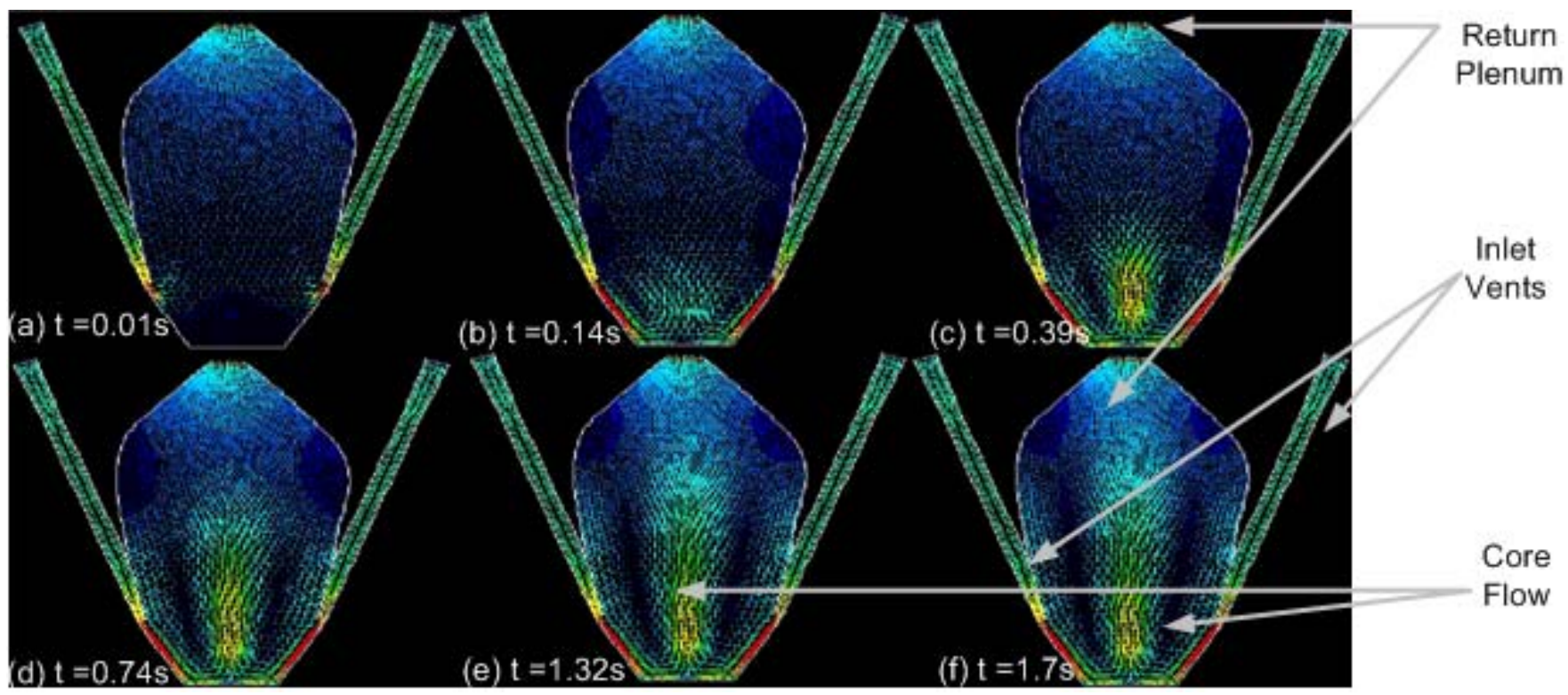

Fig. 6. Stages of stable flow development for scaled TPAWT with return plenum 
screens and causes the core flow to oscillate and destabilize as shown in Fig. 3. Hence, the return plenum is provided to limit motion of the vortices and help stabilize the flow, as shown in Fig. 6. This is also shown in the video attachment. The air received by the return plenum is fed back to the main plenum, forming a closed circuit as in Fig. 8. This is unlike the open system in $[2,3]$ and helps to further stabilize the wind flow.

The settling plenum and blower draw air from the return plenum shown in Fig. 8, indicating energy expenditure. This is akin to open loop control, since no sensor feedback is involved. Since energy is required, [5] defines this as predetermined active control, which supplements the reactive feedback controller above. A negative pressure of about 10 $\mathrm{Pa}$ was observed at the return plenum.

\section{Conditional Angular Rate-Switching (CARS) Controller}

Even with the Predetermined Active Control, oscillations of wind angle still persisted when the output feedback controller was applied. As the controller attempts to regulate wind angle, process delays in the system coupled with nonlinear energy exchange between the vortices results in rapid switching of wind angle, Fig. 9. This was not a problem in prior work since the vent velocity ratio was fixed and only the net airflow into the system was regulated in order to control wind speed at the user [2].

In order to resolve the rapid nonlinear oscillations of wind angle, we introduce the Conditional Angular Rate Switching controller, Fig. 7. If the rate of change of wind angle, $A=|\dot{e}|$, exceeds a bound $\mathrm{C}$, the controller is paused by setting the input to the integrator to zero, and thus the vent velocity ratio is maintained at a constant value. By holding the vent velocity ratio constant, momentum gradually transfers between the vortices and the wind angle variations are retarded. Once $A \leq C$, the controller is re-engaged and the wind angle is regulated by the output feedback controller.

\section{Controller Evaluation}

\section{A. Experimental Methods and Procedures}

The aforementioned control strategies were experimentally evaluated on a 1:4 scale model of the TPAWT facility, Fig. 8. The scale model incorporates actuated valves and a number

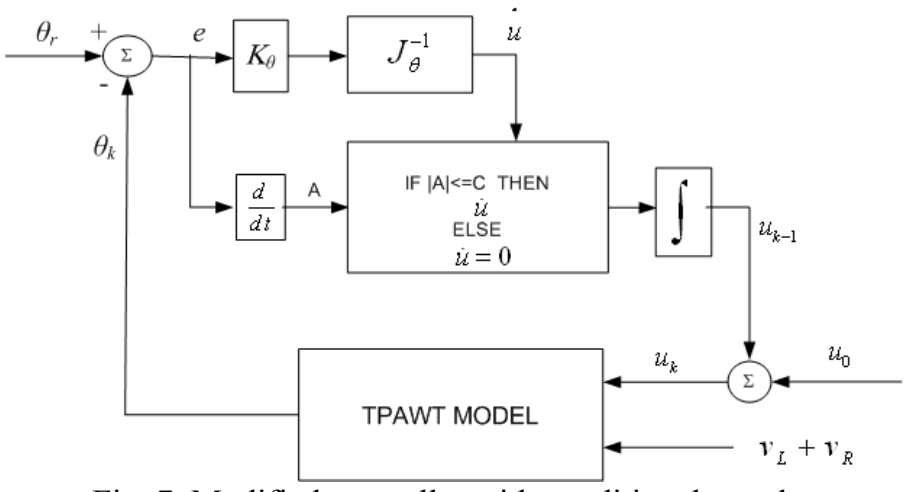

Fig. 7. Modified controller with conditional angular rate-switching control. of velocity sensors in order to facilitate real-time flow control. It is composed of a blower, a main plenum, butterfly throttling valves, ducting, and a test section. Throttling valves are mounted on the side of the plenum and are actuated by geared (66:1) Maxon DC motors (20 W).

Ducting connects the valves to the TPAWT test section as in [2]. Inlet ducting transitions smoothly from round to square ducting and leads to a long horizontal contraction. The contraction reduces from the aforementioned square cross section to a smaller rectangular cross section in order to accelerate the flow into the facility and reduce potential for backflow. The inlet vents are mounted symmetrically at an angle $\Phi$ with the test section walls. Experiments show that the flow at the user is insensitive to the vent angle $\Phi$. The test section is comprised of Lexan (side walls, ceiling) and wood

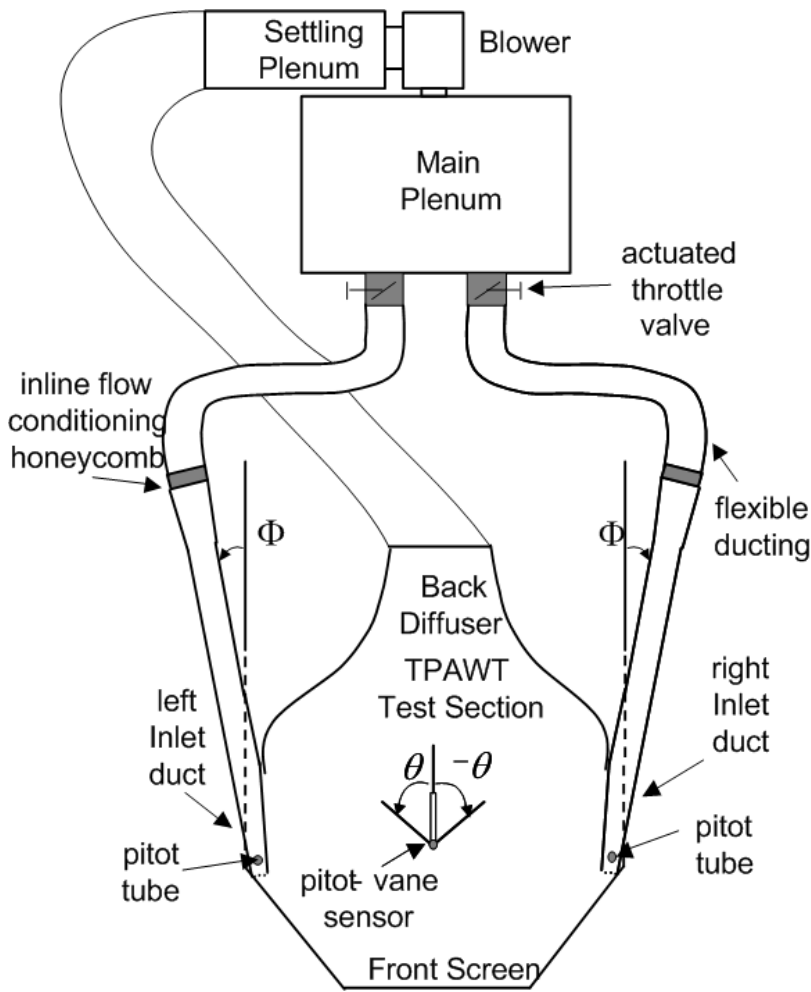

Fig. 8. Geometry of pressure plenum at the rear of the TPAWT facility

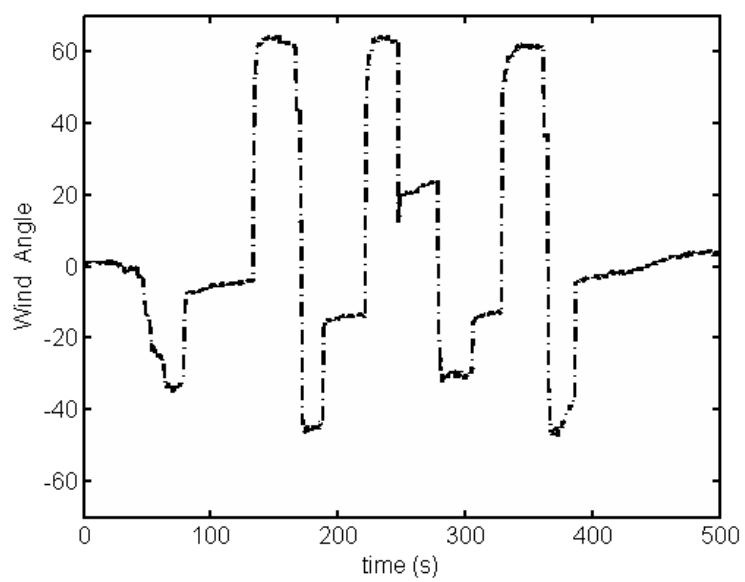

Fig. 9. Higher angular velocity causing oscillations. 
(floor). In order to approximate 2D flow, the gap height between floor and ceiling was reduced to $13 \mathrm{~cm}$ throughout the test section. Interested readers can refer [22] for further details on the design of the test bed.

The TPAWT test section has pitot tubes at each of the inlet ducts to determine vent velocities. A pitot-vane sensor mounted at the user position determines wind speed and direction at the user. The vane was designed to orient the pitot tube along the streamlines of the flow. Dwyer 607-21 Differential Pressure Transmitters with a $250 \mathrm{~ms}$ response time were used to measure pitot tube pressure. Potentiometers measure valve angles and pitot-vane angle. A dSpace 1103 Controller Board operating at a $1 \mathrm{kHz}$ sampling rate is used to control the experimental apparatus from Simulink using the Real Time Workshop toolbox. Vent velocity is regulated by PI control loops that regulate valve angles. Blower speed (e.g. fan frequency) is manually regulated before the experiments to assure that sufficient plenum pressure is available to supply adequate flow through the vents.

\section{B. Vorticity-Meter}

Although the pitot-vane measures the angle of wind at the user, we were concerned that it may be at the edge of the core flow as wind angle is regulated. Thus, a vorticity-meter was designed in order to detect proximity of the pitot-vane to the edge of the core flow.

The vorticity-meter consists of a paddle wheel mounted to an encoder directly above the pitot-vane, Fig. 10. If the wind velocity on each side of the paddle wheel is equal, the vorticity meter will not spin. The average torque acting on the two sides of the paddle cancels out. Thus, we can deduce when the pitot-vane is near the center of the core flow. The turbulent nature of the core flow, however, causes the paddle to oscillate with low amplitude without any rotations. The vorticity meter is not used for feedback, but it is used to assure that the core flow passes through the user position.

\section{Results and Discussion}

It can be seen from Fig. 11 that the flow settles to the desired angle in 40s. The numbered vertical lines illustrate the different stages of activity of the Output Feedback Controller

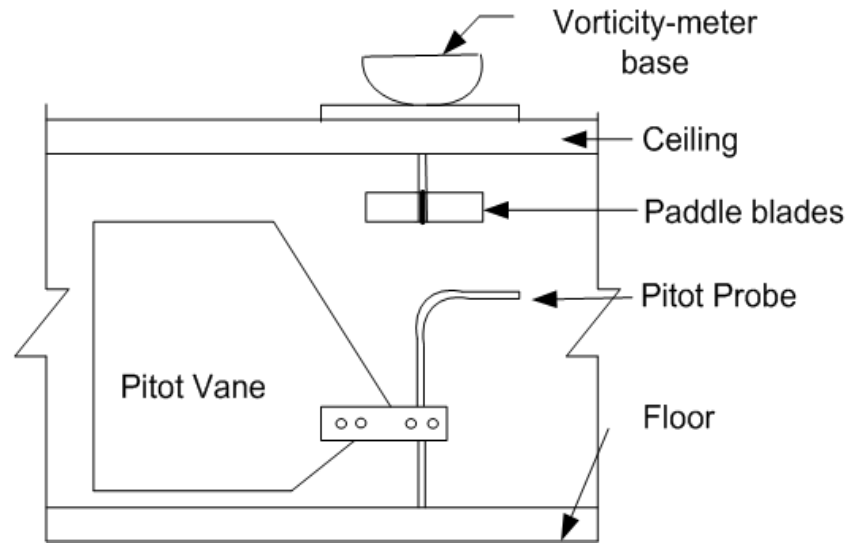

Fig. 10: Side view of vorticity-meter and Pitot-vane. and CARS controller. At Line \#1, the increase in vent velocity ratio has energized the vortices sufficiently to push the core flow towards the user. This occurs at about $34 \mathrm{~s}$. This involves the four principles of vortex motion: a) space filling, b) self-organizing c) parity adjusting and d) spectral refining as described in [23] along with time delay. Hence, there is no change in the initial pitot-vane angle for that duration.

At Line \#2 ( 36 s) the core flow begins to shift abruptly due the nonlinear exchange of energy between the vortices. Here the rate of change of wind angle, $\mathrm{A}$, has exceeded the bound $\mathrm{C}$ detailed in IV.C and the conditional angular rate-switching (CARS) controller sets $\dot{u}=0$. At Line \#3 ( $\sim 38 \mathrm{~s}), \mathrm{A}<\mathrm{C}$ such that the output feedback controller is again reactivated. At Line \#4 $(\sim 39 \mathrm{~s})$ the wind angle is within $\sim 1^{\circ}$ of the desired value.

Note that the vorticity-meter indicates angular velocity fluctuations of the wind flow. Between Lines \#1 and \#4, we see that the angular velocity fluctuation of the vorticity-meter reduces considerably. This indicates that core flow shifts away from the vorticity meter such that no wind forces are acting upon it. The core flow is then pushed back by the vortices towards the user position and the vorticity-meter. Thus, at Line \#4, the oscillations in the vorticity-meter are again apparent.
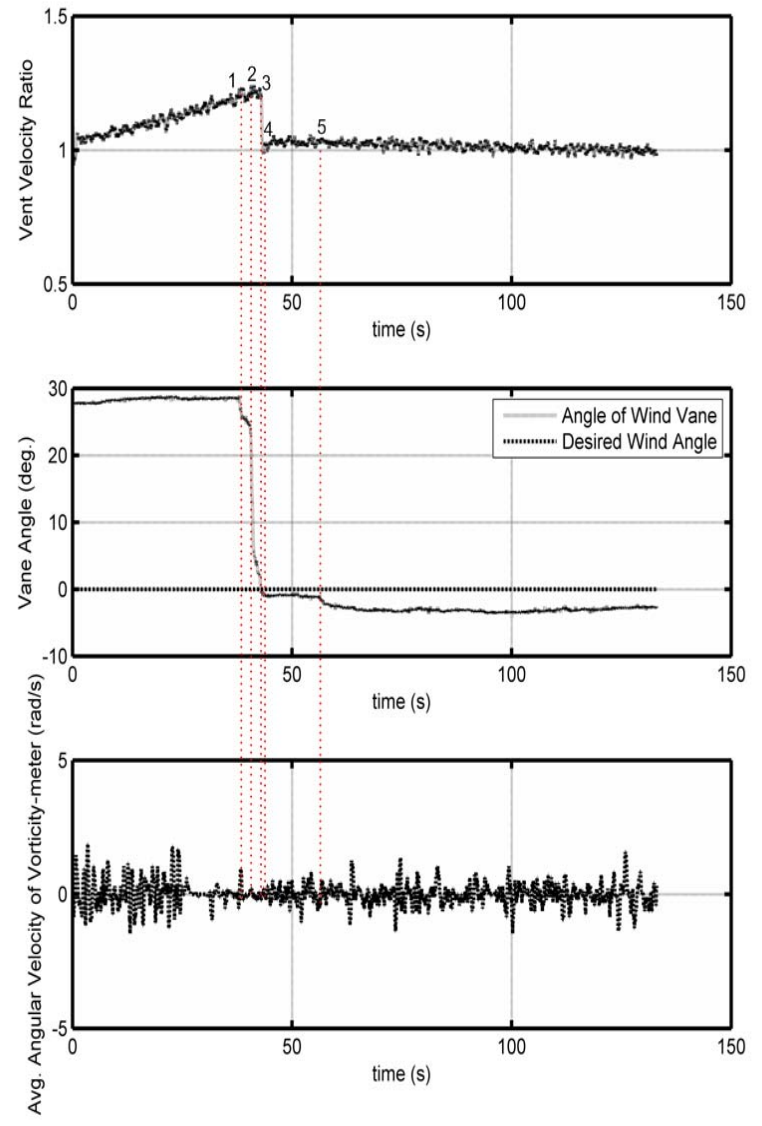

Fig. 11 Feedback control of wind angle using conditional angular rate controller and return plenum 
It can be seen at Line $\# 5$ that a slight deviation of $\sim 3^{\circ}$ results. This is attributed to shearing of flow by the vorticity-meter and the vane. The average angular velocity of the vorticity-meter is observed to be approximately $0 \mathrm{rad} / \mathrm{s}$. This indicates that flow is centered at the user position. Thus, these experiments demonstrate the ability of the system to regulate a wind heading to a quasi-steady state value within $\sim 3^{\circ}$ of the desired value. This is quite unlike [2] where an angle error of $10-15^{\circ}$ was observed, which indicates that the controllers presented in this paper provide $\sim 75 \%$ improvement in the heading angle control.

\section{Ongoing and Future work}

Although this system geometry and controller gives a higher accuracy and reduced settling time in comparison to [2], the inherent nature of the return plenum limits the controlled wind angle in the proximity of $\theta=0^{\circ}$. Ongoing work focuses on additional controller and geometric modifications to increase the range of achievable wind angles and to provide combined wind speed and angle control.

Future work will then focus on improved dynamic wind response and construction of the full scale TPAWT system. Model reduction techniques may offer improved model predictions with reduced calculation. Incorporation of such reduced models into the controller will lead to the ability to create more complex flow patterns with faster dynamics, such as gusts, crosswinds, and shear flows. Perceptual studies of wind direction will then be possible.

\section{CONCLUSION}

A method of developing steady head wind on a user in the Treadport Active Wind Tunnel (TPAWT) is presented. A negative pressure plenum is first constructed to help stabilize flow. Then a feedback control law involving output feedback of wind angle using the small gain theorem in conjunction with dynamic extension, and a conditional angular rate control, is applied. This provides accurate stable head wind at the user.

\section{ACKNOWLEDGMENT}

The authors would like to thank Charles Fisher, Meredith Metzger, Pete Willemsen, Richard Kirkman, and Hamid Sani for being part of the TPAWT group.

\section{REFERENCES}

[1] W. N. Dinh Huong Q., Song Chang, Kobayashi Akira, Hodges Larry F., "Evaluating the Importance of Multi-sensory Input on Memory and the Sense of Presence in Virtual Environments," Proceedings of the IEEE Virtual Reality, pp. 222, 1999

[2] S. D. Kulkarni, Minor, M.A., Deaver, M.W., and Pardyjak, E.R, "Output feedback control of wind display in a virtual environment," in Proc. IEEE Intl. Conf. Robotics and Automation, Rome, Italy, pp. 832 - 839 2007.

[3] R. Kirkman, M. Deaver, E. Pardyjak, and M.Metzger, "Sensitivity Analysis of a Three-Dimensional Wind Tunnel Design," in 2006 ASME Joint U.S.-European Fluids Engineering Summer Meeting, FEDSM, Miami, FL, pp. pp. 102006.
[4] J.M Hollerbach, "Locomotion interfaces," in Handbook of Virtual Environments: Design, Implementation, and Applications, e. K.M Stanley, Ed.: Lawrence Erlbaum Associates, Inc., 2002, pp. 239-254.

[5] M. Gad-el-Hak., Flow control : passive, active, and reactive flow management. Cambridge, New York: Cambridge University Press, 2000.

[6] D. Dochain, J. P. Babary, and N. Tali-Maamar, "Modeling and adaptive control of nonlinear distributed parameter bioreactors via orthogonal collocation," Automatica, vol. 28, pp. 873-883, 1992

[7] A. A. Patwardhan, G. T. Wright, and T. E. Edgar, "Nonlinear model-predictive control of distributed-parameter systems," Chemical Engineering Science, vol. 47, pp. 721-735, 1992

[8] P. K. Gundepudi and J. C. Friedly, "Velocity control of hyperbolic partial differential equation systems with single characteristic variable," Chemical Engineering Science, vol. 53, pp. 4055-4072, 1998

[9] W.H.Ray, Advanced Process Control. New York: McGraw Hill, 1981.

[10]H. Choi, R. Temam, P. Moin, and J. Kim, "Feedback control for unsteady flow and its application to the stochastic Burgers equation," Journal of Fluid Mechanics, vol. 253, pp. 509-43, 1993

[11] S. S. Joshi, J. L. Speyer, and J. Kim, "A systems theory approach to the feedback stabilization of infinitesimal and finite-amplitude disturbances in plane Poiseuille flow," Journal of Fluid Mechanics, vol. 332, pp. $157-84,1997$

[12]M. Desai and K. Ito, "Optimal controls of Navier-Stokes equations," SIAM Journal on Control and Optimization, vol. 32, pp. 1428-1446, 1994

[13]T. R. Bewley, R. Temam, and M. Ziane, "A general framework for robust control in fluid mechanics," Physica D, vol. 138, pp. 360-92, 2000

[14]P. D. Christofides and A. Armaou, "Nonlinear Control of Navier Stokes Equations," in Proceedings of the American Control Conference, Philadelphia, Pennsylvania June 1998.

[15]B. B. King and Y.-R. Ou, "Nonlinear dynamic compensator design for flow control in a driven cavity," in Proceedings of the 1995 34th IEEE Conference on Decision and Control. Part 4 (of 4), Dec 13-15 1995, New Orleans, LA, USA, pp. 3741-3746 1995.

[16]S. S. Ravindran, "A reduced-order approach for optimal control of fluids using proper orthogonal decomposition," International Journal for Numerical Methods in Fluids, vol. 34, pp. 425-48, 2000

[17]A. Chatterjee, "An introduction to the proper orthogonal decomposition," Current Science, vol. 78, pp. 808-17, 2000

[18]P. D. Christofides and A. Armaou, "Global stabilization of the Kuramoto-Sivashinsky equation via distributed output feedback control," Systems and Control Letters, vol. 39, pp. 283-294, 2000

[19]E. H. Dowell, K. C. Hall, and M. C. Romanowski, "Eigenmode analysis in unsteady aerodynamics: reduced order models," Applied Mechanics Reviews, vol. 50, pp. 371-385, 1997

[20]J. A. Burns and D. Rubio, "Distributed parameter control approach to sensor location for optimal feedback control of thermal processes," in Proceedings of the 1997 36th IEEE Conference on Decision and Control. Part 3 (of 5), Dec 10-12 1997, San Diego, CA, USA, pp. 2243-2247 1997.

[21]R. D'Andrea and G. E. Dullerud, "Distributed Control Design for Spatially Interconnected Systems," IEEE Transactions on Automatic Control, vol. 48, pp. 1478-1495, 2003

[22]M. Deaver, Pardyjak, E., Kulkarni, S., and M. Minor, "Creating Wind Display for a Virtual Environment," Journal of Fluids Engineering, 2008

[23]K. E. Gustafson, Vortex Methods and Vortex Motion. Philadelphia: SIAM, 1991 